\title{
Editorial
}

\section{Important Topics and Issues in Vascular Biology}

\author{
Timmy Lee ${ }^{*} \S$
}

\begin{abstract}
Department of Medicine, Division of Nephrology, University of Alabama at Birmingham, $17202^{\text {nd }}$ Ave. South, Zeigler Research Building 524, Birmingham, AL 35294-0007, USA
\end{abstract}

$70 \%$ of patients who reach end stage renal disease (ESRD) utilize hemodialysis as their renal replacement modality [1]. Thus, a sustainable and reliable vascular access is critical to deliver dialysis therapy to these patients. However, vascular access dysfunction remains a major cause of morbidity and mortality in the hemodialysis population. The estimated cost of treating vascular access dysfunction in the United States annually is approximately one billion dollars [2]. The primary reasons for vascular access dysfunction are the high proportion of arteriovenous fistulas (AVF) that fail to mature and frequent stenosis in arteriovenous grafts (AVG), resulting in prolonged dialysis catheter use [3-5]. Currently, there are few, if any, effective therapies to treat vascular access dysfunction. This is in large part because of the poor understanding of the pathophysiology of vascular access dysfunction, particularly vessel remodeling and neointimal hyperplasia development.

To expand the discussion of hemodialysis vascular access dysfunction, this issue of the The Open Urology and Nephrology Journal will focus on several important topics and issues in vascular biology as it relates to hemodialysis vascular access dysfunction. The topics have been carefully chosen to highlight important subjects in the biology of vascular access, which have not been extensively reviewed and addressed in the literature.

First, the review by Rotmans provides a very detailed and eloquent description of the role of animal models to improve the understanding of the pathophysiologic mechanisms of vascular access dysfunction. His review focuses on both large and small animal models of hemodialysis vascular access, and their respective advantages and disadvantages. Appropriate animal models will be crucial to identify new pathways that are important to neointimal hyperplasia development and vascular remodeling for therapeutic targets, and testing of novel therapeutic interventions and devices.

The age of the ESRD population continues to slowly rise [1]. Increased conduit artery conduit artery stiffness is a key factor in the pathogenesis of aging-associated diseases and

*Address correspondence to this author at the Department of Medicine, Division of Nephrology, University of Alabama at Birmingham, $17202^{\text {nd }}$ Ave. South, Zeigler Research Building 524, Birmingham, AL 35294-0007, USA; Tel: 205-975-9322; Fax: 205-996-6465; E-mail: txlee@uab.edu

${ }^{\S}$ Guest Editor progressive CKD. A major factor leading to arterial stiffness is development of vascular calcification. In this current issue Lee sheds light on the pathophysiology of vascular calcification in CKD and ESRD. His review further expands the topic of vascular calcification and discusses its importance in hemodialysis vascular access dysfunction. In addition, Paulson in his review expands on the importance of vascular function and provides an extensive and comprehensive review of vascular function and elasticity and clinical significance as it relates to hemodialysis vascular access dysfunction.

Hemodynamic shear stresses after AV access creation and the response of the endothelium to these new shear stresses play an important role in AV access remodeling and neointimal hyperplasia development [3, 4]. This clinical and research area of hemodynamics in arteriovenous access has been an area of emerging cutting edge research. One potential area for future therapies is to modify hemodynamic shear stress patterns to promote improved endothelial response. Fitts et al. in their review discusses the novel methods we have to-date to calculate hemodynamic profiles in AV access in experimental and clinical models and biological factors that may modulate the impact of hemodynamic shear stresses on vascular remodeling.

Lastly, it is well known that we lack effective treatment therapies for vascular access dysfunction. Kumbar et al. provide an excellent overview of the current therapies to treat and prevent vascular access dysfunction. Furthermore, they also discuss the use of novel paradigms that may be useful to predict future vascular access dysfunction and used as tools for improving vascular access selection.

The biology of vascular access dysfunction is an emerging area of scientific research in the nephrology field. The advent of new and effective therapies will be contingent upon an improved understanding of the biologic mechanisms that promote arteriovenous fistula and graft failure. However, clinical management of vascular access dysfunction will always still be an art. In the future, the art will be choosing a combination of novel therapies that fit's each patient's underlying biology and individualized access planning and selection.

\section{REFERENCES}

[1] Collins AJ, Foley RN, Herzog C, et al. US Renal data system 2012 annual data report. Am J Kidney Dis 2013; 61: A7, e1-476. 
[2] Feldman HI, Kobrin S, Wasserstein A. Hemodialysis vascular access morbidity. J Am Soc Nephrol 1996; 7: 523-35.

[3] Lee T, Roy-Chaudhury P. Advances and new frontiers in the pathophysiology of venous neointimal hyperplasia and dialysis access stenosis. Adv Chronic Kidney Dis 2009; 16: 329-38.
[4]

Lee T. Novel Paradigms for dialysis vascular access: Downstream vascular biology-Is there a final common pathway? Clin J Am Soc Nephrol 2013; 8(12): 2194-201.

[5] Roy-Chaudhury P, Sukhatme VP, Cheung AK. Hemodialysis vascular access dysfunction: A cellular and molecular viewpoint. J Am Soc Nephrol 2006; 17: 1112-27.

(C) Timmy Lee; Licensee Bentham Open.

This is an open access article licensed under the terms of the Creative Commons Attribution Non-Commercial License (http://creativecommons.org/licenses/by-nc/3.0/) which permits unrestricted, non-commercial use, distribution and reproduction in any medium, provided the work is properly cited. 